\title{
Efeito da Isotretinoína na Xerostomia, pH e Fluxo Salivar
}

\section{The Effect of Isotretinoin on Xerostomia, pH and Flow Salivary}

\author{
Ana Paula Martins Gomes ${ }^{\text {**; }}$ Cristiane Vasconcellos Caspar Nobre ${ }^{\mathrm{b}}$; Gildete Costa Drumond Bento ${ }^{\mathrm{c}}$; \\ Antonio Augusto Gomes ${ }^{\mathrm{d}}$; Ana Maria Martins Gomes ${ }^{\mathrm{e}}$; Renata Oliveira Guaréa
}

\author{
aUniversidade Cruzeiro do Sul, Programa de Pós-Graduação Stricto Sensu em Odontologia. São Paulo, SP. Brasil. \\ bodontovix, Consultório Odontológico. Praia da Costa. Vila Velha, ES

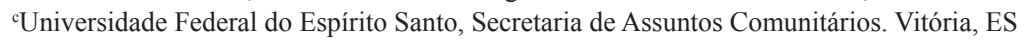 \\ dUniversidade Federal do Espírito Santo, Departamento de Prótese Dentária Santa Luzia Vitória - ES \\ eUniversidade Federal do Espírito Santo, Departamento de Clínica Odontológica. Vitória, ES \\ *E-mail: anapaulamgomes@hotmail.com \\ Recebido em: 17/08/2015; Aceito em: 18/11/2015
}

\begin{abstract}
Resumo
A isotretinoína é um derivado sintético da vitamina A empregada no tratamento da acne. As reações adversas na cavidade bucal são pouco relatadas na literatura. Esta pesquisa consiste em um estudo transversal que teve como objetivo avaliar a influência da isotretinoína no grau de xerostomia, pH e fluxo salivar. Após aprovação pelo CEP do Hospital Infantil Nossa Senhora da Glória - SESA, e consentimento e/ou assentimento dos participantes, foi aplicado um questionário, o Xerostomia Inventory Dutch version, para avaliar o grau de xerostomia e coletada a saliva total para análise do fluxo e $\mathrm{pH}$. A amostra foi constituída por 87 indivíduos, divididos em quatro grupos: G1 - controle, indivíduos não acneicos saudáveis ( $\mathrm{n}=16)$; $\mathrm{G} 2$ - indivíduos acneicos que nunca fizeram uso de isotretinoína ( $\mathrm{n}=16$ ); G3- indivíduos acneicos em uso de isotretinoína $(n=45)$; G4- indivíduos acneicos que haviam concluído o tratamento com isotretinoína (n=10). Os indivíduos deste último grupo haviam concluído o tratamento em um intervalo de 30 a 60 dias. Com base nos dados obtidos foram calculados média, mediana, desvio padrão e aplicado o teste estatístico de Kruskal Wallis. Os resultados dos parâmetros estudados para os grupos G1, G2, G3 e G4 foram, respectivamente: grau de xerostomia - 16,44 $\pm 3,16,17,62 \pm 2,68,21,55 \pm 3,45,18,00 \pm 2,71$ ( $<0,0001$ ); fluxo salivar $-1,08 \pm 0,40,0,89 \pm 0,37$, $0,90 \pm 0,58$ e $1,22 \pm 0,55 \mathrm{~mL} / \mathrm{min}$ ( $\mathrm{p}=0,139)$; $\mathrm{pH}$ da saliva $-7,05 \pm 0,37,7,05 \pm 0,32,6,89 \pm 0,24,6,91 \pm 0,24$ ( $\mathrm{p}=0,104)$. Concluiu-se que o fluxo salivar foi menor nos indivíduos acneicos que não usam e os que usam a isotretinoína (G2 e G3) em relação aos grupos G1 e G4, porém esta diferença não foi estatisticamente significante. A xerostomia nos indivíduos acneicos que usam a isotretinoína (G3) foi maior em relação aos grupos G1, G2 e G4 e esta diferença foi estatisticamente significante.
\end{abstract}

Palavras-chave: Isotretinoína. Xerostomia. Acne Vulgar.

\begin{abstract}
Isotretinoin is a synthetic derivative of vitamin A, used in the treatment of acne. The adverse reactions in the oral cavity are scarcely reported in the literature. This research consists of a cross-sectional study in healthy and aims to evaluate the influence of isotretinoin in the degree of xerostomia, salivary flow and pH. After approval by the IRB at Children's Hospital Nossa Senhora da Glória-SESA, individuals have signed the informed consent form, and the search was performed by applying a questionnaire, Xerostomia Inventory Dutch version (XID), to assess the degree of xerostomia and collecting overall saliva for flow analysis and pH. The sample consisted of 87 subjects, divided into four groups: G1 Control - no acneicos, healthy individuals ( $n=16)$; 22 acneicos individuals who have never made use of isotretinoin $(n=$ 16); G3 acneicos individuals using isotretinoin ( $n=45)$; G4 acneicos individuals who had completed treatment with isotretinoin ( $n=10)$. Individuals of this latter group had completed treatment in a range of 30 to 60 days. From the obtained values were calculated the average, standard deviation and applied a statistical test of Kruskal Wallis. The results of the parameters studied for G1, G2, G3 and G4 were: degree of xerostomia-16.44 $\pm 3.16,17.62 \pm 2.68,21.55 \pm 3.45,18.00 \pm 2.71$ ( $p<0.0001)$; salivary flow $-1.08 \pm 0.40,0.89 \pm 0.37,0.90 \pm 0.58$, and $1.22 \pm 0.55$ $\mathrm{mL} / \mathrm{min}(\mathrm{p}=0.139)$; saliva $\mathrm{pH}-7.05 \pm 0.37,7.05 \pm 0.32,6.89 \pm 0.24,6.91 \pm 0.24(p=0.104)$. It was found that the salivary flow was higher in acneicos individuals who do not use and those who use isotretinoin (G2 and G3) in relation to the groups G1 and G4, but this difference was not statistically significant. Xerostomia in acneicos individuals who use isotretinoin (G3) was higher compared to the G1, G2 and G4 and this difference was statistically significant.
\end{abstract}

Keywords: Isotretinoin. Xerostomia. Acne Vulgaris.

\section{Introdução}

A acne é uma doença de pele autolimitada e multifatorial que acomete os folículos sebáceos de seres humanos. A introdução da isotretinoína a partir de 1982 representou uma conquista no tratamento sistêmico dos casos mais graves de acne, a cística e a modular. Atualmente a isotretinoína tem sido indicada para outros tipos de acne menos graves, como a rosácea, em casos de acne com cicatriz, ou nos casos de acne que não responde à terapia convencional, bem como para tratamento de outras condições não-acneicas. Entretanto, devido às controvérsias de seu uso, ao potencial teratogênico e às várias reações adversas seu uso deve ser feito com cautela ${ }^{1-8}$.

A Secretaria de Saúde do Espírito Santo - SESA distribui este fármaco gratuitamente para a população por meio da Farmácia Cidadã. A inserção dos indivíduos no protocolo de tratamento com isotretinoína obedece à orientação do 
Ministério da Saúde ${ }^{7}$. Este preconizou o uso da isotretinoína para tratamento da acne em uma dose de 0,5-2,0 mg/kg/ dia. Como critérios de exclusão adotados estão gravidez, amamentação e hipersensibilidade à isotretinoína. Seu uso deve ser evitado ou realizado com cuidado em indivíduos com: menos de 15 anos; dificuldades de compreender e executar as orientações médicas; insuficiência hepática; alterações no metabolismo de lipídios. Os indivíduos ainda precisam realizar o controle da dosagem da transaminase hepática durante o tratamento ${ }^{7}$.

As reações adversas envolvendo o uso de isotretinoína estão comumente relacionadas à pele/membranas mucosas e aos sistemas nervoso, músculo esquelético, linfático, gastrintestinal, cardiorrespiratório e geniturinário ${ }^{2}$. Máformação fetal em mulheres grávidas e alterações em órgãos ou em sistemas também são relatadas ${ }^{3}$. Com relação à cavidade bucal, há relatos de que o índice de CPO-d (dentes cariados, perdidos e obturados) aumentou significativamente e o fluxo salivar diminuiu gradualmente com o tempo ${ }^{9}$. Xerostomia, queilite angular e hipossalivação ${ }^{10}$, além de secura labial, são também efeitos adversos do uso da isotretinoína ${ }^{3}$.

As estruturas mineralizadas e os tecidos moles presentes na cavidade bucal são banhados pela saliva, produto de secreção das glândulas salivares e do fluido crevicular gengival ${ }^{11,12}$. A saliva é um fluido biológico clinicamente informativo, que é útil para abordagens no prognóstico, diagnóstico laboratorial e/ou clínico, no monitoramento e manejo de pacientes com doenças sistêmicas e bucais, tais como a doença periodontal e a cárie ${ }^{13-15}$. As múltiplas funções da saliva estão relacionadas com sua composição e sua característica fluida ${ }^{11,12}$.

A xerostomia é definida como sintoma subjetivo de boca seca, e a hipofunção da glândula salivar refere-se à hipossalivação. O primeiro só pode ser avaliado por meio de um questionário estruturado e o segundo, por avaliação do fluxo salivar ${ }^{16}$. A xerostomia é um problema comum e multifatorial. O sintoma pode ser em razão da redução da quantidade de saliva produzida ou de uma mudança na composição da saliva, mas a sensação de boca seca pode também estar presente em pessoas com produção normal de saliva $^{17}$.

A xerostomia é uma condição subjetiva que só pode ser avaliada por um questionamento direto ao pacientes ${ }^{18}$. O Xerostomia Inventory - XI consiste em um questionário fechado, com questões diretas, que avalia o grau subjetivo de xerostomia ${ }^{19}$. O XI consiste de onze itens que representam os aspectos comportamentais de indivíduos com xerostomia, com cinco opções de respostas. O questionário Xerostomia Inventory Dutch version - XID consiste em uma forma simplificada com apenas três opções de $\operatorname{respostas}^{20}$. As respostas compreendem em: nunca (1 ponto), ocasionalmente ( 2 pontos) e sempre ( 3 pontos). O valor obtido para cada indivíduo é calculado somando-se todos os pontos. A pontuação pode variar de 11 a 33 pontos e quanto mais próximo de 33, maior é o grau de xerostomia.

A saliva é um material de pesquisa amplamente estudado, em virtude da sua importância na manutenção da saúde bucal ${ }^{21}$. Tendo em vista os aspectos acima relatados e a escassez de informações com relação aos efeitos adversos da isotretinoína, esta pesquisa tem como objetivo avaliar a influência do uso desse medicamento na ocorrência de xerostomia, $\mathrm{pH}$ e fluxo salivar.

\section{Material e Métodos}

Esta pesquisa consiste em um estudo analítico observacional transversal. A pesquisa foi aprovada pelo Comitê de Ética do Hospital Infantil Nossa Senhora da Glória - SESA (Protocolo n ${ }^{\circ}$ 11/2011). Participantes maiores de 18 anos assinaram Termo de Consentimento Livre e Esclarecido - TCLE; os menores de 18 anos assinaram o Assentimento Informado, tendo o TCLE sido assinado pelo seu responsável.

A amostra foi composta por indivíduos saudáveis para o grupo controle e indivíduos que tiveram indicação de isotretinoína para tratamento da acne e que procuraram a Farmácia Cidadã Metropolitana-SESA, localizada em Cariacica-ES, para receber o fármaco gratuitamente. Eles foram informados da existência deste estudo, ficando a critério sua participação na pesquisa. A amostra foi dividida em: G1- controle, indivíduos não acneicos saudáveis ( $\mathrm{n}=16$, 5 do sexo feminino e 11 do masculino); G2- indivíduos acneicos que nunca fizeram uso de isotretinoína $(n=16,5$ do sexo feminino e 11 do masculino); G3- indivíduos acneicos em uso de isotretinoína ( $\mathrm{n}=45,15$ do sexo feminino e 30 do masculino); G4- indivíduos acneicos que haviam concluído o tratamento com isotretinoína $(n=10,5$ do sexo feminino e 5 do masculino). Os indivíduos deste último grupo concluíram o tratamento com isotretinoína num intervalo de 30 a 60 dias.

A amostra foi estratificada adotando como variável dependente a faixa etária (15-30 anos). Todos os participantes do sexo feminino faziam uso de anticoncepcional oral. Foram adotados como critérios de exclusão indivíduos que na entrevista relataram: ser tabagistas, consumir álcool regularmente, possuir alguma doença sistêmica ou fazer uso de medicamentos que alteram o fluxo salivar (antidepressivo, antipsicótico, antiansiolítico, anti-hipertensivo, antihistamínico, opióide e multimedicação).

Os participantes foram submetidos à anamnese para coleta de dados referentes à identificação, histórico odontológico e médico. Também, foi aplicado o questionário XID (Quadro 1) e em seguida, foi realizada a coleta da saliva total dos participantes e a leitura do seu $\mathrm{pH}$. Em uma ficha clínica, foram anotados os dados e os resultados dos exames realizados. A coleta da saliva foi realizada em um consultório odontológico, entre 8:00 e 10:00 horas da manhã, em ambiente bem iluminado e com temperatura média de $23{ }^{\circ} \mathrm{C}$. Os participantes foram orientados a não se alimentar, não ingerir qualquer tipo de líquido e não escovar os dentes num período de 1 hora e 30 
minutos antes da coleta.

Quadro 1: Questionário aplicado aos participantes da pesquisa

\begin{tabular}{|c|l|c|}
\hline & \multicolumn{1}{|c|}{ Itens } & Pontuação \\
\hline 1 & $\begin{array}{l}\text { Bebe um pouco de líquido para ajudar na } \\
\text { deglutição dos alimentos }\end{array}$ & \\
\hline 2 & Sente a boca seca durante as refeições & \\
\hline 3 & Levanta à noite para tomar líquidos & \\
\hline 4 & Sente a boca seca & \\
\hline 5 & Tem dificuldade em comer alimentos secos & \\
\hline 6 & $\begin{array}{l}\text { Chupa balas ou guloseimas para aliviar a } \\
\text { boca seca }\end{array}$ & \\
\hline 7 & $\begin{array}{l}\text { Tem dificuldades para deglutir certos } \\
\text { alimentos }\end{array}$ & \\
\hline 8 & Sente a pele da face seca & \\
\hline 9 & Sente os olhos secos & \\
\hline 10 & Sente os lábios secos & \\
\hline 11 & Sente o interior do meu nariz seco & \\
\hline & & Total \\
\hline
\end{tabular}

Fonte: Van der Putten et $a l .{ }^{21}$.

Após 30 minutos de permanência dos participantes no ambiente refrigerado, sentados confortavelmente, com a cabeça ereta e os olhos abertos, foi realizada a coleta da saliva. Os participantes foram orientados a mastigar um pedaço de mangueira de látex atóxico, estéril, de tamanho padronizado de $1 \mathrm{~cm}$ de comprimento e $0,5 \mathrm{~cm}$ de diâmetro com uma frequência de 60 ciclos/minuto, alternando o lado da mastigação. No primeiro minuto, toda a saliva produzida foi deglutida. Após o primeiro minuto, os participantes foram orientados a expectorar a saliva dentro de um copo graduado de $15 \mathrm{~mL}$ (Lena ${ }^{\circledR}$ Ind. Bras.). Utilizando um relógio (Quartz ${ }^{\circledR}$ ), o tempo de coleta de 5 minutos foi cronometrado.

O volume da saliva foi medido ao final da coleta da saliva, utilizando o próprio copo graduado. Para eliminação das bolhas de ar presentes na saliva coletada, foi utilizada uma espátula $\mathrm{n}^{\circ} 24$ (Duflex®) banhada em álcool absoluto (Emfal ${ }^{\circledR}$ Ind. Bras.). Para calcular o fluxo salivar, o volume final da saliva secretada foi dividido pelo tempo da coleta. A velocidade de secreção foi expressa em mililitro por minuto ( $\mathrm{mL} / \mathrm{min}$ ). $\mathrm{O} \mathrm{pH}$ foi medido eletrometricamente no local da coleta após avaliação do fluxo salivar, utilizando o pHmetro modelo 3510 (JENWAY®)

Para análise descritiva, os dados obtidos foram lançados em planilha do pacote estatístico IBM SPSS Statistics, versão 20.0, sendo calculados a média aritmética e o desvio padrão. Para análise estatística foi aplicado o teste de Kruskal Wallis, nível de significância de $5 \%(0,05)$. Foram construídos gráficos box plot que apresentam os valores máximo, mínimo, mediana, primeiro quartil, e terceiro quartil, além de outliers.

\section{Resultados e Discussão}

A amostra foi constituída por 87 pacientes, sendo $34,48 \%$ $(n=30)$ do sexo feminino e $65,52 \%(n=57)$ do masculino. A idade média de todos os indivíduos foi de 20,36 $\pm 3,8$ anos; do sexo feminino foi de $21,8 \pm 4,7$ anos e do masculino $19,7 \pm 2,9$ anos. A dose de isotretinoína utilizada pelos indivíduos para o tratamento da acne foi de 0,33-1,09 mg/ $\mathrm{kg} / \mathrm{dia}$.

Os resultados obtidos com o XID, fluxo e $\mathrm{pH}$ da saliva estão apresentados na Tabela 1 e Gráficos 1 a 3. O grau de xerostomia para os grupos G1, G2, G3 e G4 foram, respectivamente, $\quad 16,44 \pm 3,16, \quad 17,62 \pm 2,68, \quad 21,55 \pm 3,45$, $18,00 \pm 2,71$. O grupo controle (G1) apresentou menor grau de xerostomia, e o grupo de indivíduos acneicos que estavam utilizando isotretinoína (G3) apresentou o maior. É importante observar que o G4, composto por indivíduos acneicos que haviam terminado o tratamento com isotretinoína, apresentou grau de xerostomia menor do que o G3. Esta diferença foi estatisticamente significante (Tabela 1 e Gráfico 1).

Tabela 1: Grau de xerostomia, fluxo ( $\mathrm{ml} / \mathrm{min})$ e $\mathrm{pH}$ da saliva de acordo com os grupos estudados

\begin{tabular}{ccccc}
\hline Grupos & N & XID & Fluxo Salivar & Ph da Saliva \\
\hline G1 & 16 & $16,44 \pm 3,16$ & $1,08 \pm 0,40$ & $7,05 \pm 0,37$ \\
G2 & 16 & $17,62 \pm 2,68$ & $0,89 \pm 0,37$ & $7,05 \pm 0,32$ \\
G3 & 45 & $21,55 \pm 3,45$ & $0,90 \pm 0,58$ & $6,89 \pm 0,24$ \\
G4 & 10 & $18,00 \pm 2,71$ & $1,22 \pm 0,55$ & $6,91 \pm 0,24$ \\
$p$-valor & & $<0,0001$ & 0,139 & 0,104 \\
\hline *p $<0,05$ & & & &
\end{tabular}

Fonte: Dados da pesquisa.

Gráfico 1: Representação gráfica dos valores médios, mediana e desvios padrões dos graus de xerostomia dos grupos estudados.

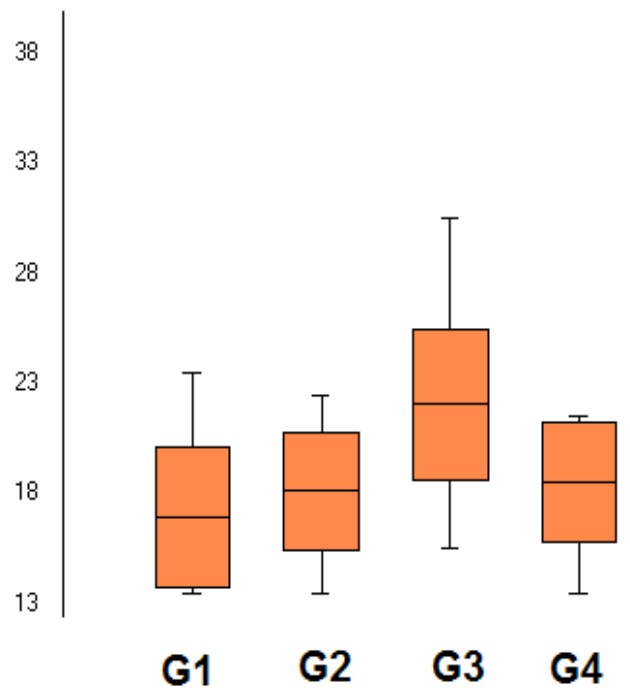

Fonte: Dados da pesquisa.

O fluxo salivar para os grupos G1, G2, G3 e G4 foram, respectivamente, $1,08 \pm 0,40,0,89 \pm 0,37,0,90 \pm 0,58$, e $1,22 \pm 0,55 \mathrm{~mL} / \mathrm{min}$., não sendo observada diferença estatisticamente significante entre os grupos (Tabela 1 e Gráfico 2). O grupo de indivíduos acneicos que não usa isotretinoína (G2) e o grupo de indivíduos acneicos que usa isotretinoína (G3) apresentaram valores de fluxo salivar muito próximo com presença de outliers, que evidenciam valor acima, afetando os valores médios e o desvio padrão (Tabela 1 e Gráfico 2). 
Gráfico 2: Representação gráfica dos valores médios, mediana, desvios padrões do fluxo salivar dos grupos estudados

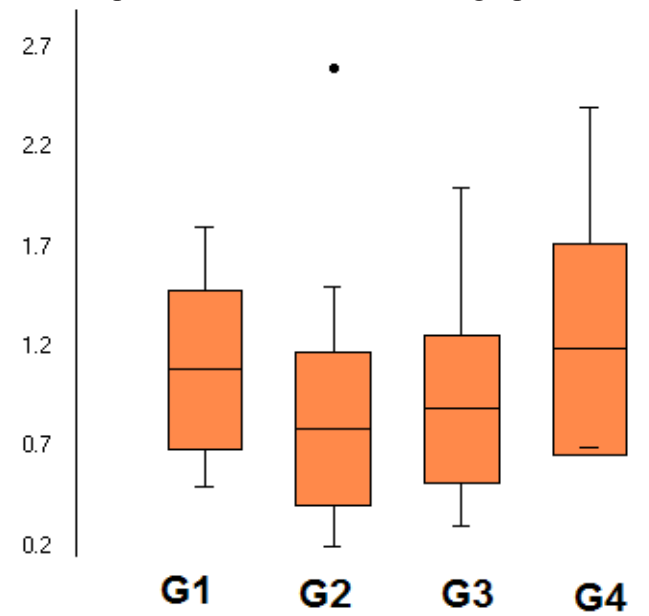

Fonte: Dados da pesquisa.

Com relação ao $\mathrm{pH}$ da saliva, os valores obtidos para os grupos G1, G2, G3 e G4 foram, respectivamente: 7,05 $\pm 0,37$, $7,05 \pm 0,32,6,89 \pm 0,24,6,91 \pm 0,24$ (Tabela 1 e Gráfico 3). Os valores para este parâmetro foram semelhantes e não apresentaram diferenças estatisticamente significantes.

Gráfico 3: Representação gráfica dos valores médios, mediana e desvios padrões do $\mathrm{pH}$ da saliva dos grupos estudados

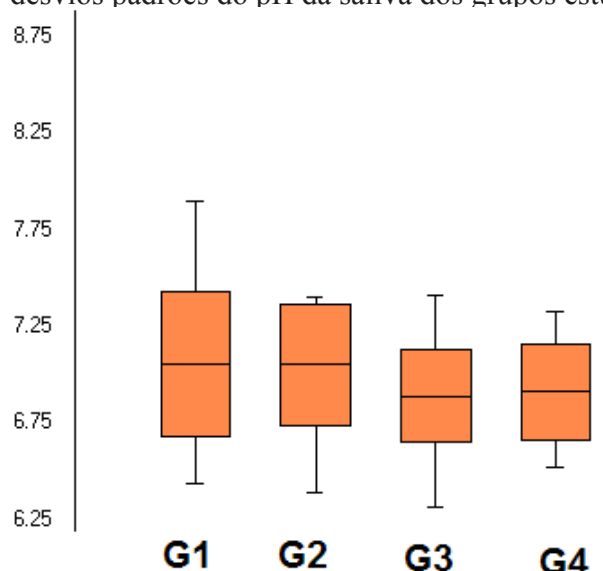

Fonte: Dados da pesquisa.

Para avaliar o grau de xerostomia dos indivíduos desta pesquisa foi utilizado o $\mathrm{XID}^{20}$. Os resultados obtidos demonstraram um aumento no grau da xerostomia nos indivíduos que usam isotretinoína (Gráfico 1). Os dados foram analisados pelo teste de Kruskal Wallis, e os resultados foram estatisticamente significantes $(<0,0001)$, ou seja, nos indivíduos acneicos em uso de isotretinoína, o grau de xerostomia é maior do que nos indivíduos do grupo controle, não acneicos saudáveis, e nos indivíduos acneicos que fizeram uso de isotretinoína (Tabela 1). Analisando os dados obtidos para esse grupo, pode-se inferir que após o término do tratamento, o grau de xerostomia diminuiu, retornando aos valores normais.

Considerando as respostas ao XID pelos participantes deste estudo, as principais regiões que sofreram ressecamento pelo uso da isotretinoína $(0,4-1,0 \mathrm{mg} / \mathrm{kg} / \mathrm{dia})$ foram os lábios, a pele da face, os olhos e a mucosa do nariz ressecados. Estes achados são semelhantes aos relatos de outros autores. Gomes et $a l .{ }^{10}$, por exemplo, relataram que todos os indivíduos que utilizaram isotretinoína na dose de $0,5-0,7 \mathrm{mg} / \mathrm{kg} / \mathrm{dia}$, por um período de no mínimo um mês, apresentaram xerostomia. Windhorst e Nigra ${ }^{22}$ foram os primeiros a publicar um trabalho que relatou alterações nos tecidos bucais (boca seca e queilite) provocadas pelo uso da isotretinoína, que era utilizada em uma dose $109 \mathrm{mg} /$ dia. Nesta ocasião afirmaram que a boca seca foi relatada em $30 \%$ dos indivíduos que utilizaram isotretinoína.

Os trabalhos relacionados com a análise da saliva em pacientes que usaram isotretinoína são poucos. Neste estudo, foi observado um fluxo salivar menor com uma média de $0,90 \pm 0,58$ e $0,89 \pm 0,37 \mathrm{~mL} / \mathrm{min}$ para os indivíduos acneicos usuários (G2) e não usuários (G3) de isotretinoína, respectivamente. Após o término do tratamento (30 a 60 dias), o fluxo salivar apresentou uma média de 1,22 $\pm 0,55$ $\mathrm{mL} / \mathrm{min}$, valor relativamente maior em comparação ao grupo controle, indivíduos não acneicos saudáveis $1,08 \pm 0,40 \mathrm{~mL} /$ min. A diferença entre estes valores não foi estatisticamente significante (Tabela 1). Este resultado não é semelhante ao de outros autores que encontraram diminuição do fluxo salivar em pacientes usuários de isotretinoína ${ }^{9,10,23}$. Porém, Reynolds et $a l .{ }^{23}$ não observaram alteração no fluxo salivar. Assim como neste estudo, Lupi-Pégurier et al. ${ }^{9}$ observaram que os pacientes recuperaram o fluxo salivar dois meses após o fim do tratamento. Entretanto Bots, van Nieuw Amerongen e Brand $^{24}$ relataram um caso de hipossalivação irreversível com três anos de acompanhamento, e o correlacionaram com o uso da isotretinoína.

$\mathrm{O}$ pH da saliva apresentou uma ligeira diminuição com o uso da isotretinoína, porém não foi significante (Tabela 1). Na literatura, apenas Oikarinen et al. ${ }^{16}$ avaliaram a variação do $\mathrm{pH}$, e o resultado obtido foi semelhante ao deste estudo.

De acordo com Hopcraft e $\operatorname{Tan}^{18}$, a xerostomia pode estar ou não acompanhada por redução no fluxo salivar. Toida et al..$^{25}$ constataram que $44,4 \%$ dos indivíduos que relatam a sensação de boca seca apresentam fluxo salivar normal. Furness et al. ${ }^{17}$ afirmaram que a xerostomia pode estar associada à redução da quantidade de saliva produzida, ou à mudança na composição da saliva, mas que indivíduos com fluxo salivar normal podem apresentar esse sintoma.

O mecanismo de ação da isotretinoína na glândula sebácea ainda não está totalmente esclarecido, mas sabe-se que dentre os efeitos terapêuticos da isotretinoína incluem a redução do tamanho das glândulas sebáceas em até $90 \%$, a redução da atividade das glândulas sebáceas e a diminuição da produção de sebo ${ }^{26-28}$. Örsal et al. ${ }^{29}$ avaliaram o efeito da isotretinoína na função das glândulas salivares usando a cintilografia e observaram diferenças significativas após o terceiro e o sexto mês de tratamento, em comparação com os valores obtidos antes do tratamento. A cintilografia mostrou claramente que as funções das glândulas parótidas e submandibulares foram 
afetadas pela isotretinoína.

Assim como uma série de critérios de exclusão/inclusão é adotada pelo Ministério da Saúde para o uso da isotretinoína no tratamento da $\mathrm{acne}^{7}$, e diante das evidências da influência dessa droga na saúde bucal, é de fundamental importância ressaltar o papel do cirurgião dentista como membro da equipe multiprofissional no acompanhamento desses pacientes.

\section{Conclusão}

Com base nos resultados obtidos foi possível concluir que o fluxo salivar foi menor nos indivíduos acneicos que não usam e nos que usam a isotretinoína (G2 e G3) em relação aos grupos ao G1 e G4, porém esta diferença não foi estatisticamente significante. A xerostomia nos indivíduos acneicos que usam a isotretinoína (G3) foi maior em relação aos grupos G1, G2 e G4 e esta diferença foi estatisticamente significante.

\section{Referências}

1. Balducci-Roslindo E, Silvério KG, Jorge MA, Gonzaga HF. Effect of isotretinoin on tooth germ and palate development in mouse embryos. Braz Dent J 2001;12:115-9.

2. Diniz DGA, Lima EM, Antoniosi Filho NR. Isotretinoína: perfis farmacológico, farmacocinético e analítico. Braz J Pharm Sci 2002;38:415-30.

3. Silva Júnior ED, Sette IMF, Belém LF, Janebro DI, Pereira GJS, Barbosa JAA, et al. Isotretinoína no tratamento da acne: riscos x benefícios. Rev Bras Farm 2009;90:186-9.

4. Nickle SB, Peterson N, Peterson M. Updated physician's guide to the off-label uses of oral isotretinoína. J Clin Aesthet Dermatol 2014;7:22-34.

5. Rigopoulos D, Larios G, Katsambas AD. The role of isotretinoin in acne therapy: why not as first-line therapy? facts and controversies. Clin Dermatol 2010;28:24-30.

6. Korting HC, Schollmann C. Current topical and systemic approach to treatment of rosacea. JEADV 2009;23:876-82.

7. Brasil. Ministério da Saúde. Protocolo de uso da isotretinoína no tratamento da acne grave. Brasília: Portaria $n^{\circ} 1.159$ de 18 de novembro de 2015. [acesso em 13 jul 2015]. Disponível em http://portalsaude.saude.gov.br/images/ pdf/2015/novembro/20/PT--SAS-PCDT-Acne-GraveATUALIZADO-10-11-2015.

8. Sinclair W. The rational use of systemic isotretinoin in acne: a call for moderation. S Afr Med J 2012;102:282-4.

9. Lupi-Pégurier L, Muller-Bolla M, Fontas E, Ortonne JP. Reduced salivary flow induced by systemic isotretinoin may lead to dental decay. A prospective clinical study. Dermatology 2007;14:221-6.

10. Gomes SGF, Custódio W, Cury AA, Garcia RC. Effect of salivary flow rate on masticatory efficiency. Int J Prosthodont 2009;22:168-72.
11. Dawes C. Circadian rhythms in human salivary flow rate and composition. J Physiol 1972;220:529545.

12. Edgar WM. Saliva: its secretion, composition and functions. Br Dent J 1992;172:305-12.

13. Schipper RG, Silletti E, Vingergoeds MH. Saliva as research material: Biochemical, physicochemical and practical aspects. Arch Oral Biol 2007;52:1114-35.

14. Spielmann N, Wong DT. Saliva: diagnostics and therapeutic perspectives. Oral Dis 2011;17:345-54.

15. Malamud D. Saliva as a diagnostic fluid. Dent Clin North Am 2011;55:159-78.

16. Oikarinen K, Salo T, Kylmäniemi M, Palatsi R, Karhunen T, Oikarinen A. Systemic oral isotretinoin therapy and flow rate, $\mathrm{pH}$, and matrix metalloproteinase-9 activity of stimulated saliva. Acta Odontol Scand 1995;53:369-71.

17. Furness S, Worthington HV, Bryan G, Birchenough S, Mcmillan R. Interventions for the management of dry mouth: topical therapies. Cochrane Database Syst Rev 2012;5.

18. Hopcraft MS, Tan C. Xerostomia: an update for clinicians. Aust Dent J 2010;55:238-44.

19. Thomson WM, Chalmers JM, Spencer AJ, Williams SM. The Xerostomia inventory: a multi-item approach to measuring dry mouth. Community Dent Health 1999;16:12-7.

20. Van Der Putten GJ, Brand HS, Schols Jos MGA, Baat Cl. The diagnostic suitability of a xerostomia questionnaire and the association between xerostomia, hyposalivation and medication use in a group of nursing home residents. Clin Oral Investig 2011;15:185-92.

21. Pedersen AM, Bardow A, Jensen SB, Nauntofte B. Saliva and gastrointestinal functions of taste, mastication, swallowing and digestion. Oral Dis. 2002;8:117-29.

22. Windhorst DB, Nigra T. General clinical toxicicology of oral retinoids. J Am Acad Dermatol. 1982;6:675-82.

23. Reynolds NJ, Gough M, Clamp JR, Burton JL. Effect of oral isotretinoin therapy on saliva volume and composition. Bras J Dermatol. 1991;125:89-90.

24. Bots CP, Van Nieuw Amerongen A, Brand HS. Blijvend en droge mond na behandeling van acne. Ned Tijdschr Tandheelkd. 2003;110:295-7.

25. Toida M, Nanya Y, Takeda-Kawaguchi T, Baba S, Iida K, Kato $\mathrm{K}$, et al. Oral complaints and stimulated salivary flow rate in 1188 adults. J Oral Pathol Med 2010;39:407-19.

26. Lestringant GG, Frossard PM, Agarwal M, Galadari IH. Variations in lipid and lipoprotein levels during isotretinoin treatment for acne vulgaris with special emphasis on HDLcholesterol. Int J Dermatol 1997:36:859-62.

27. Zouboulis CC. Isotretinoin revisited: pluripotent effects on human sebaceous gland cells. J Invest Dermatol 2006:126:2154-6.

28. Brelsford M, Beute T. Preventing and Managing the side effects of isotretinoin. Semin Cutan Med Surg 2008;27:197206.

29. Örsal E, Seven B, Erdem MT, Varoglu E, Farimaz H, Ayan AK. Turk J Med Sci Evaluation of the effect of isotretinoína on salivary gland function by Tc-99m pertechnetate imaging in acne vulgaris patients. 2015;43:674-7. 